\title{
STRONGLY DRAZIN INVERSE IN RINGS
}

\author{
Huanyin Chen, Seyed Saeed Nabavi And Marjan Sheibani Abdolyousefi
}

Abstract. An element $a \in R$ has strongly Drazin inverse if there exists $a^{\prime} \in R$ such that $a a^{\prime}=$ $a^{\prime} a, a^{\prime}=a^{\prime} a a^{\prime}$ and $a-a a^{\prime} \in R$ is nilpotent, i.e., $a$ is strongly nil-clean. Additive results for strongly Drazin inverse in a ring are presented. The explicit formulas for such generalized inverse of $a+b$ are given. These extend the results on s-Drazin and Drazin inverses of Wang (Filomat, 31(2017), 1781-1789), Yang and Liu (J. Comput. Appl. Math., 235(2011), 1412-1417). As an application, we give various conditions under which a $2 \times 2$ block matrix has s-Drazin inverse. Mathematics subject classification (2010): 15A09, 32A65, 16E50.

Keywords and phrases: Drazin inverse, additive property, block matrix, spectral idempotent.

\section{REFERENCES}

[1] C. Bu And C. Zhang, A note on the formulas for the Drazin inverse of the sum of two matrices, Linear Algebra Appl., 439(2013), 565-579.

[2] H. Chen, On strongly nil clean matrices, Comm. Algebra, 41(2013), 1074-1086.

[3] J. Chen, Z. XU AND Y. WeI, Representations for the Drazin inverse of the sum $P+Q+R+S$ and its applications, Linear Algebra Appl., 430 (2009) 438-454.

[4] A. S. Cvetkovic and G. V. Milovanovic, On the Drazin inverse of operator matrices, J. Math. Analysis Appl., 375(2011), 331-335.

[5] A. J. DiESL, Nil clean rings, J. Algebra, 383(2013), 197-211.

[6] D. Djordjevic And Y. WeI, Additive results for the generalized Drazin inverse, J. Austral. Math. Soc., 73 (2002), 115-125.

[7] D. S. Ilic Cvetrovic And Y. Wei, Algebraic Properties of Generalized Inverses, Developments in Mathematics 52. Springer, Singapore, 2017.

[8] J. Ji AND Y. WEI, The Drazin inverse of an even-order tensor and its application to singular tensor equations, Comput. Math. Appl., 75 (2018), 3402-3413.

[9] J. J. Koliha, A generalized Drazin inverse, Glasgow. Math .J., 38(1996), 367-381.

[10] M. T. Kos AN, Z. WANG AND Y. ZHOU, Nil-clean and strongly nil-clean rings, J. Pure Appl. Algebra, 220(2015), 633-646.

[11] J. Ljubis aVlueviC And D. S. CvetkoviC-Illic, Additive results for the Drazin inverse of block matrices and applications, J. Compt. Appl. Math., 235(2011), 3683-3690.

[12] D. Mosic, Reverse order laws for the generalized strongly Drazin inverses, Appl. Math. Comput., 284(2016), 37-46.

[13] P. PAtricio And R. E. Hartwig, Some additive results on Drazin inverses, Appl. Math. Comput., 215(2009), 530-538.

[14] S. QIAO, X. WANG AND Y. WEI, Two finite-time convergent Zhang neural network models for timevarying complex matrix Drazin inverse, Linear Algebra Appl., 542 (2018) 101-117.

[15] S. QIAO AND Y. WeI, Acute perturbation of Drazin inverse and oblique projectors, Front. Math. China, 13 (2018) 1427-1445.

[16] Z. WANG, A class of Drazin inverses in rings, Filomat, 31(2017), 1781-1789.

[17] G. Wang, Yimin Wei And S. QiaO, Generalized Inverses: Theory and Computations, Second edition, Developments in Mathematics, 53. Springer, Singapore; Science Press Beijing, Beijing, 2018.

[18] Y. WEI, Acute perturbation of the group inverse, Linear Algebra Appl., 534 (2017), 135-157.

[19] Y. M. Wei And C. Y. Deng, A note on additive results for the Drazin inverse, Linear Multilinear Algebra, 59(2011), 1319-1329. 
[20] H. YAnG, X. F. LiU, The Drazin inverse of the sum of two matrices and its applications, J. Comput. Appl. Math., 235(2011), 1412-1417. 